\title{
Potential therapeutic road for targeting the SARS-CoV-2 at throat
}

\author{
Kaya $\mathrm{M}^{1}$, Abuaisha $\mathrm{AM}^{1}$, Serakinci $\mathrm{N}^{2,3}$, Ozturk $\mathrm{S}^{1}$ \\ Department of Medical Genetics, Internal Medicine, Istanbul University Istanbul Faculty of Medicine, \\ Istanbul, Turkey. kmurat@istanbul.edu.tr
}

\begin{abstract}
INTRODUCTION: Coronavirus disease 2019 (COVID-19) has become a serious public health problem for 183 out of 197 countries in the world. Understanding the routes and pathogenesis of the coronavirus is important and it is considered that the studies on host cell receptor Angiotensin Converting Enzyme 2 (ACE2) may be valuable for the treatment and prevention of the disease.

AIM: To evaluate the possibility of inhibition of SARS-CoV-2 at throat.

METHODS: A comprehensive literature search was conducted.

CONCLUSION: In view of the fact that the mouth and nose have higher number of ACE2 expressed cells, they serve as a gateway for the virus to enter. Thus, blocking the gate could be a good choice to reduce or even prevent the transmission. Small interfering RNAs (siRNAs) are double-stranded RNA molecules and could be designed easily and directed against many strains of a virus. Due to their features, siRNAs can provide a potential strategy to interfere with the replication of viral diseases. We think that since oral and nasal epithelial cells are relatively easily accessible it may allow to develop siRNA molecules to inhibit SARS-CoV-2 already at the entry where it continues to replicate for a period (Fig. 1, Ref. 50). Text in PDF www.elis.sk KEY WORDS: SARS-CoV-2, COVID-19, ACE2, Throat and siRNA.
\end{abstract}

\section{Introduction}

Human coronaviruses are a wide family of viruses that typically induce mild to severe upper respiratory diseases in people, such as common cold. Viruses can also invade the lower airways and cause severe illnesses including pneumonia or bronchitis (1). Coronaviruses have single-stranded RNAs of about 26,000 to 32,000 bases in length (2).

In Wuhan-China, December 2019, for the first time a cluster of atypical pneumonias associated with a novel coronavirus was detected (3). Coronavirus disease, termed COVID-19, was caused by severe acute respiratory syndrome coronavirus 2 (SARS-CoV-2; previously termed 2019-nCoV), which can spread from person to person (4).

SARS-CoV-2 is a member of the Coronaviridae family and includes a number of viruses that cause the common cold (5).

${ }^{1}$ Department of Medical Genetics, Internal Medicine, Istanbul University Istanbul Faculty of Medicine, Istanbul, Turkey, ${ }^{2}$ Department of Medical Genetics, Near East University Faculty of Medicine, Nicosia, North Cyprus, Mersin, Turkey, and ${ }^{3}$ Department of Molecular Biology and Genetics, Faculty of Art and Sciences, Near East University, Nicosia, North Cyprus, Mersin, Turkey

Address for correspondence: M. Kaya, Dr, Istanbul University, Istanbul Faculty of Medicine, Division of Internal Medicine, Department of Medical Genetics, Millet street. 34390 Capa - Istanbul, Turkey.

Phone: +90.212 .4142000$
Coronaviruses have been identified in many hosts including cam- els, bats, masked palm civets, mice, dogs, and cats (6). Most of coronaviruses that are pathogenic to humans cause mild clinical symptoms (7) with two notable exceptions: severe acute respiratory syndrome coronavirus (SARS-CoV), the other is Middle East respiratory syndrome coronavirus (MERS-CoV). SARS-CoV is a novel beta-coronavirus discovered first in China in November 2002 (8) and resulted in more than 8000 human infections and 774 deaths in 37 countries during 2002-03 (9). SARS-CoV-2 was first defined using next-generation sequencing in infected individuals with typical symptoms, including high fever, dyspnea, and radiographs showing invasive lesions in both lungs $(10,11)$.

Real-time reverse transcription polymerase chain reaction (qRT-PCR) test is used in the molecular biology laboratories for the diagnosis of COVID-19. This test is used for the qualitative detection of nucleic acid from SARS-CoV-2 in upper and lower respiratory specimens such as nasopharyngeal or oropharyngeal swabs, sputum, lower respiratory tract aspirates, bronchoalveolar lavage, and nasopharyngeal wash/aspirate or nasal aspirate. These specimens are collected from individuals suspected of COVID-19 by their healthcare provider (12). In addition to qRT-PCR, computed tomography (CT) scans and routine blood tests are used for the diagnosis of COVID-19. The effectiveness of existing antibody tests to diagnose active COVID-19 infections and to define whether individuals with SARS-CoV-2-positive antibodies are protected from re-infection is unknown (13). 


\section{Entry of SARS-CoV-2 into the Body}

COVID-19 can be a mild upper respiratory tract disease at first. The primary transmission of the SARS-CoV-2 occurs by droplet transmission. Respiratory secretions of infected cases have vital role for release of the virus. The mucosal surfaces such as nose, mouth and eyes are the first entry gates of the SARS-CoV-2 to the body (14).

In Wolfel et al study most of patients appeared to be above their peak in the upper respiratory tract samples when they were first tested, while the sputum shedding of infectious virus continued during the first week of symptoms (15). Diagnostic testing of viral RNA, antibody response with clinical correlation suggests that viral RNA remains positive over three weeks despite the mild symptoms or full resolution of symptoms (15).

In line with viral load work from Wolfel et al (15) additional recent studies indicated the ability of using saliva as a diagnostic sample, in two studies on coughed out saliva 11 out of 12 and 20 out of 23 showing positive results in SARS-CoV-2 infected patients. This indicates ability of using saliva sample as a diagnostic sample for the identification of SARS-CoV-2 infection. Saliva sample can be collected through coughing out, saliva swabs and directly from salivary gland duct. Despite this fact early diagnosis of COVID-19 by using saliva specimen is still limited but promising since especially the sample collection process is less invasive than nasal or throat swab and safer for health care workers (16). Additionally, it allows us to focus on targeting the virus replication at the time of the presence in the throat.

KK et al (16) 2020 tested the S gene of SARS-CoV-2 by using qRT-PCR in saliva specimens and found that 11 out of 12 patients were positive $(91.67 \%) .33$ patients who were negative for the laboratory analysis were also negative for the saliva examination. In another study from the same group that used self-collected saliva from deep throat by COVID-19 patients and tested for SARSCoV-2 RNA. Specimens were a mixture of nasopharyngeal and bronchopulmonary secretions from deep throat by coughing out in the morning. 20 out of 23 COVID-19 patients showed positive results for SARS-CoV-2 RNA (16). Also, after the treatment, in this group SARS-CoV-2 RNA was detected in saliva, even if using antibodies against SARS-CoV-2, viral RNA could still be detected for 20 days or even longer in deep throat saliva specimens of one third of patients. This suggests that the viral RNA could stay for a long period instead of dying out after antibody application. Interestingly, one of COVID-19 patients with completely resolved symptoms was found SARS-CoV-2 RNA positive again after 2 days of negative results, suggesting that low levels of SARS-CoV-2 RNA could still be excreted in saliva even after clinical recovery (16).

Furin is an enzyme highly expressed in lung tissue $(17,18)$ and has been implicated in the infection of SARS-CoV-2 (19). Furin-like cleavage site in the S protein of SARS-CoV-2 has been identified $(18,20)$. Tongue can serve as a high risk route for SARSCoV-2 entry simply due to high expression of ACE2 in the oral cavity. Besides, "theoretically" it suggests that cells expressing furin have lower restriction for entry of the virus. Also, the role of furin-like cleavage site in SARS-CoV-2 infection needs to be studied in more detail (17).

In addition to the common symptoms of COVID19, some patients have been reported to experience problems with their sense of smell and taste (21). We speculate that this situation could be due to the infected cells related to smell and taste in the nose and mouth. One of the other entry gates of the virus to the body is nose. It has been reported that nose goblet and ciliated cells have increased ACE2 and TMPRSS2 levels and it has been highlighted that nasal carriage is possibly an important feature of transmission, therefore, some intranasally administered drugs and vaccines could limit the transmission effectively (22).

\section{Entry of the SARS-CoV-2 into the Cell}

Cellular entry of SARS-CoV depends on spike (S) protein binding to a specific cellular receptor (23). As in SARS-CoV, SARS-CoV-2 enters the cells through binding to ACE2 receptor and the affinity of this binding is a major determinant of SARS$\mathrm{CoV}$ replication rate and disease severity (24). Viral entry also depends on TMPRSS2 protease activity and cathepsin B/L activity may substitute for TMPRSS2 (25). Elevated expression of ACE2 and TMPRSS2 have been reported in alveolar epithelial type II cells (22). Also, by using immunohistochemistry, ACE2 and TMPRSS2 have been detected in both nasal and bronchial epithelium (26). Cells in certain tissues overexpressing ACE2 like upper esophagus and stratified epithelial cells, lung alveolar type II (AT2), renal proximal tubular cells, myocardial cells and bladder urothelial cells, are known to have a large possible risk for SARS-CoV-2 infection (27). ACE2 is a master host cell receptor for SARS-CoV-2 and has a critical function for the virus to penetrate and kill the cell. Studies have shown that ACE2 is expressed in the oral cavity mucosa and is also extremely enriched in the epithelial cells of the tongue (27).

Oral cavity ACE2 expressing cells are potential risk route for SARS-CoV-2 entry, on the other hand, it may have a very critical role for prevention of the virus replication and thus will have a vital role in the treatment of the disease (27).

\section{SARS-CoV-2 threats the world}

According to the latest data, the world population is about 7.6 billion and as of 25 June 2020, there have been 9,542,451 coronavirus infected cases, 485,280 deaths and 5,186,690 recovered cases (28). Social isolation is recommended in many countries to protect against virus infection (29). This method is not sustainable due to both economic and social problems. Therefore, protection from the viral infection is much more urgent for now. Some face masks are used to prevent mouth and nose from the virus infection, however, face masks provide limited protection against coronavirus infection (30).

Many drugs are currently being studied in ongoing clinical trials, however few of them, including chloroquine, have demonstrated both in vitro and clinical antiviral efficiency in cases infected with SARS-CoV-2 (31). Unfortunately, thousands of new 


\section{6-211}

cases are being added to the number of COVID19 patients day by day. We hope that successful results can be obtained from these valuable clinical trials but till now the success in COVID19 treatment is limited (32). In addition to these ongoing clinical trials, further studies should be done urgently to effectively inhibit viruses.

\section{Prevention from the COVID-19}

The replication of the virus in the upper respiratory tract can be important for disease development as well as it can be a hope for patient's treatment and prevention from the virus (15). SARSCoV-2 virus enters host cells by ligating the spike protein (S glycoprotein) with the TMPRSS2 protease-primed host cell receptor ACE2. The ACE2-and TMPRSS2-mediated cell entry could be inhibited by experimental and proven drugs. Replication and assembly of the SARS-CoV-2 may also be blocked by antivirals targeting viral RNA-dependent RNA polymerase (RdRP) and key protease (Mpro) (33). In light of the current knowledge, nasal and mouth tissue cells may play an important role for targeting SARS-CoV-2 virus.

\section{Could SARS-CoV-2 be targeted with siRNA in Mouth or Nose?}

RNA interference (RNAi) discovered in the late 1990s as a well-known technique for silencing or suppressing target genes related with virulence and pathogenesis. Small interfering RNA (siRNA) molecules with length of approximately 20-25 base pairs are non-coding RNAs and involved in the RNAi pathway which has a natural cellular defense against RNA viruses. They interfere with the expression of target specific genes by degrading mRNAs (34).

\section{How does siRNA work}

siRNA molecule complements with the target mRNA molecule and once the siRNA enters the host cell, it is incorporated into the RNA-induced silencing complex (RISC) which leads to its activation. The sense strand is separated from the duplex and degraded by nuclease enzymes in the RISC complex that is activated while the anti-sense strand directs the RISC to the target mRNA located inside the cell cytoplasm. The mRNA binding to the antisense strand triggers the cleavage of the endonuclease Argonaut and therefore the post-transcriptional silencing of the gene (35).

\section{Chemical Modification of siRNA}

The biological characteristics of siRNAs contain negative charge, high molecular weight, and plasma instability, which render them prone to enzymatic degradation (36). To resolve this issue, some chemical modifications of siRNA have been developed to maximize stabilization, improve sensitivity and potency and escape from the immune response (37). Some of the modifications involve the incorporation of 2-O-methyl variations in the sugar structure of chosen nucleotides inside both the anti-sense and sense strands of the siRNA. The siRNA efficacy may also be improved via conjugation of small molecules or peptides to the sense strand of siRNA. Many other modifications have been revealed to minimize off-target effects, like phosphorothioate or boranophosphate introduction $(38,39)$.

\section{Viral and Non-viral delivery system of siRNAs}

Since siRNAs are hydrophilic, large sized (about $13.3 \mathrm{kDa}$ ) highly negatively charged macromolecules, they are not able to cross the biological membranes. Delivery of siRNAs to their target cells can be through viral or non-viral systems. Using viral vectors have previously demonstrated to be proper for cell uptake with high efficacy. But it has limitations for human therapeutic delivery like immunogenicity, toxicity, uncontrolled viral replication, and tumorigenicity (40). Due to these limitations, more effective and successful delivery systems are used which are non-viral delivery systems like naked siRNA delivery and delivery vectors such as lipids, polymers, peptides, and inorganic materials (35).

Naked siRNA delivery is simply the delivery of siRNA molecules without the use of a delivery vehicle or carrier. This way of delivery has many advantages including ease of preparation and facility of delivery by inhalation, intranasal or intratracheal routes. However, it faces a major problem in cell targeting and uptake and it can be degraded within the airways (39). Lipid-based delivery systems like liposomes or lipid nanoparticles are favored methods especially for inhaled pulmonary delivery. Polymer-based siRNA delivery systems that consist of biocompatible polymers may be chemically changed to enhance cellular delivery. Naked siRNA delivery hav- ing low clinical efficiency and bioavailability is enhanced with peptide-based delivery vectors. Inorganic-based delivery systems allow for the unique delivery of siRNAs via direct conjugation or non-covalent encapsulation (39).

Treatment of diseases with siRNAs has long been a very promising method and also many recent delivery systems have reached the clinical trial phase efficiently (41). In clinics, the first ever siRNA drug Onpattro (patisiran) (Alnylam) infusion for the treatment of adult patients with peripheral nerve disease (polyneuropathy) caused by inherited amyloidosis-mediated transthyretin (hATTR) was approved by the U.S. Food and Drug Administration (FDA) in August 2018 (42).

The greatest challenge to achieve potent siRNA therapy is not the design of it, but rather the way biologically active siRNAs are delivered to the target tissue. Diverse siRNA delivery methods have been developed and used in different studies (41). There are different routes for delivering siRNA categorized as local and systemic delivery (43). Several tissues or organs, like nose, oral cavity, or vagina, could be targeted for local siRNA use and specific modifications may also be made for siRNA formulations (44). Local delivery has been a desirable and an effective path, allowing reduced doses to be used and reducing adverse effects (45).

Wu et al (46) previously showed that siRNA molecules could inhibit the SARS-CoV spike sequences by targeting 3'-UTR in 


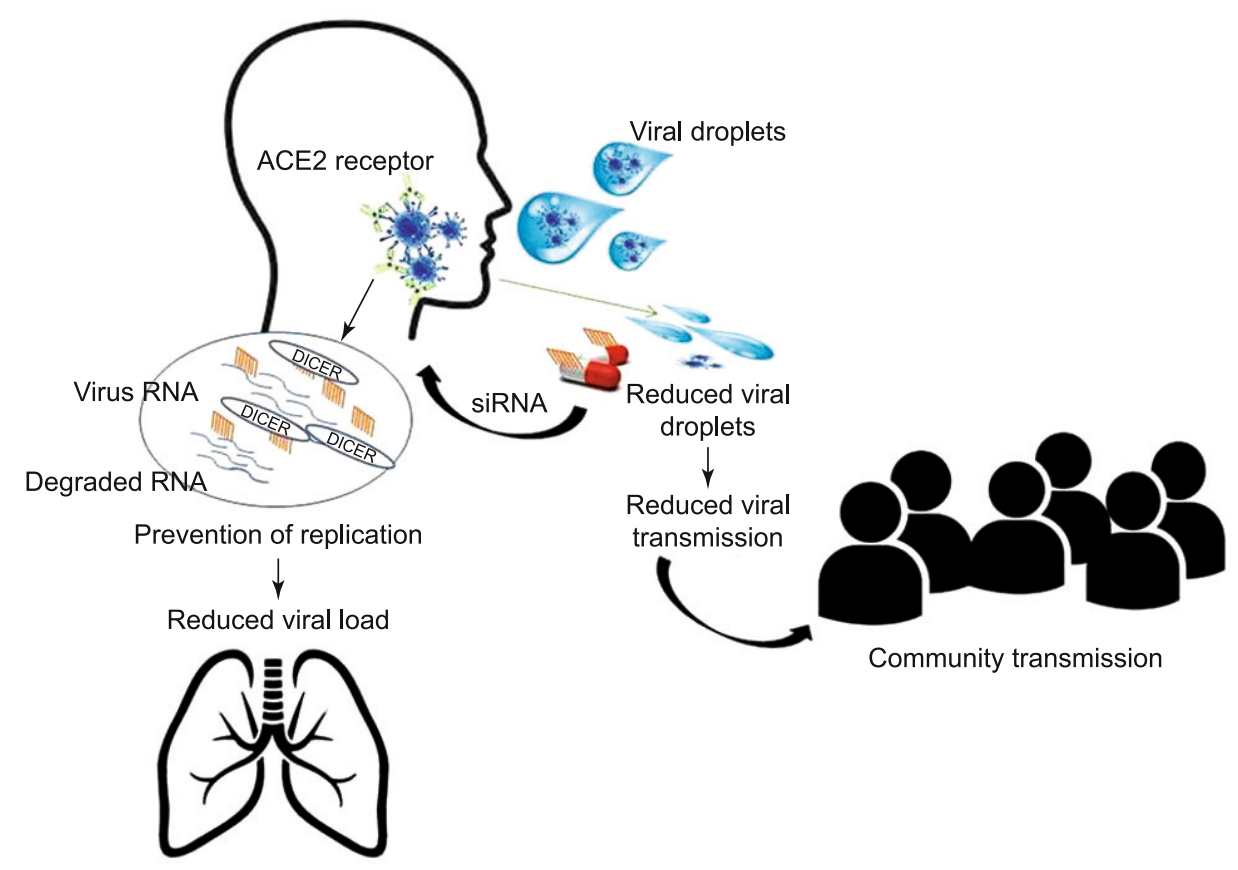

Fig. 1. Schematic representation of targeting SARS-CoV-2 virus already at upper respiratory tract by using siRNA approach to reduce viral load.

Vero-E6 cells. There have been 35 patented RNAi studies associated with anti-sars coronavirus therapy and 28 of them are about siRNA molecules (34).

To the best of our knowledge, there have been no studies documenting the effects of siRNAs on SARS-CoV-2 viruses. However, recently Alnylam company has started a promising study about the aerosolized delivery of siRNA molecules for lungs and 350 siRNA molecules have been synthesized targeting SARS-CoV2; in vitro and in vivo testing will be conducted by Vir biotechnology (47). Theoretically, it can be considered that siRNAs may be a good option for the treatment of lungs that are the most affected organ from the SARS-CoV-2. Lungs have some useful characteristics like a well perfused $(5 \mathrm{l} / \mathrm{min})$ surface area or a very thin epithelium $(0.1-0.2 \mu \mathrm{m})$ for therapeutic approaches with siRNA molecules. Nevertheless, the structure of the airways (extensively branched), existence of lung fluids like mucus and surfactant and also mucociliary clearance mechanisms containing alveolar macrophages and other difficulties could prevent the delivery of the siRNA molecules to lung cells (48). Due to these obstacles, in order for siRNA molecules to enter the target cells, siRNAs have to pass many difficult barriers yet developing new treatment methods using siRNA molecules that target the virus early as within first weeks of infection, while the virus still is at throat, will be beneficial. Even though there are some obstacles to the delivery of siRNAs that need to be overcome, we suppose that inhibition of SARS-CoV2 viruses can be achieved if siRNA delivery systems could be developed for mouth and nose which are more readily available and having limited enzymatic activity $(49,50)$.

\section{Conclusion}

There are a variety of candidate drugs which can inhibit SARS$\mathrm{CoV}-2$ infection and replication. These drugs consist of TMPRSS2 and ACE2 inhibitors. Blocking ACE2 protein and inhibition of TMPRSS2 can prevent SARS-CoV-2 from entering cells (33). Given that ACE2 and TMPRSS2 proteins are also expressed in cells in the mouth and nose, it may be beneficial to consider this inhibitor studies to destroy the SARS-CoV-2 virus.

We hereby suggest that developing an effective method that can stop the viruses in the mouth and nose before going further down the internal organs such as lungs and also this method could reduce viral transmission as we show in Figure 1. The oral cavity has limited enzymatic activity and comparatively neutral $\mathrm{pH}$ of nearly $6.2-7$. Oral mucosa and nasal mucosa have relatively easily accessible to manipulation feature $(49,50)$. Due to these characteristics, mouth and nose could be suitable for siRNA based treatments and potential ACE2 and TMPRSS2 inhibitors in COVID-19 disease. Developing potential ACE2 and TMPRSS2 in- hibitors or siRNA molecules against SARS-CoV-2 in mouth and nose cells may have some important advantages: Target inhibitors can reach cells in a shorter time even applied at low doses. Drug side effects affecting other organs can be reduced. Thus, viral load can be effectively reduced. Furthermore, this will be a supportive approach to current COVID-19 therapies. More specifically, reducing the viral load in upper respiratory tract will reduce the spread of the virus to other individuals dramatically. 


\section{References}

1. Petrosillo N, Viceconte G, Ergonul O, Ippolito G, Petersen E. COVID-19, SARS and MERS: are they closely related? Clin Microbiol Infect 2020; 26 (6): 729-734. DOI: 10.1016/j.cmi.2020.03.026.

2. Smith EC, Denison MR. Coronaviruses as DNA wannabes: a new model for the regulation of RNA virus replication fidelity. PLoS Pathog 2013; 9 (12): e1003760. DOI: 10.1371/journal.ppat.1003760.

3. Chen N, Zhou M, Dong X, Qu J, Gong F, Han Y et al. Epidemiological and clinical characteristics of 99 cases of 2019 novel coronavirus pneumonia in Wuhan, China: a descriptive study. Lancet 2020; 395 (10223): 507-513. DOI: 10.1016/s0140-6736 (20)30211-7.

4. Li H, Liu SM, Yu XH, Tang SL, Tang CK. Coronavirus disease 2019 (COVID-19): current status and future perspectives. Int J An- timicrob Agents 2020; 55 (5): 105951. DOI: 10.1016/j.ijantimicag. 2020.105951.

5. Corman VM, Muth D, Niemeyer D, Drosten C. Hosts and Sources of Endemic Human Coronaviruses. Adv Virus Res 2018; 100: 163-188. DOI: 10.1016/bs.aivir.2018.01.001.

6. Cavanagh D. Coronavirus avian infectious bronchitis virus. Vet Res 2007; 38 (2): 281-297. DOI: 10.1051/vetres:2006055.

7. Su S, Wong G, Shi W, Liu J, Lai ACK, Zhou J et al. Epidemiology, Genetic Recombination, and Pathogenesis of Coronaviruses. Trends Microbiol 2016; 24 (6): 490-502. DOI: 10.1016/j.tim.2016.03.003.

8. Peiris JS, Guan Y, Yuen KY. Severe acute respiratory syndrome. Nat Med 2004; 10 (Suppl 12): S88-97. DOI: 10.1038/nm1143.

9. Chan-Yeung M, Xu RH. SARS: epidemiology. Respirology 2003; 8 Suppl (Suppl 1): S9-14. DOI: 10.1046/j.1440-1843.2003.00518.x.

10. Zhu N, Zhang D, Wang W, Li X, Yang B, Song $\mathbf{J}$ et al. A Novel Coronavirus from Patients with Pneumonia in China, 2019. N Engl J Med 2020; 382 (8): 727-733. DOI: 10.1056/NEJMoa2001017.

11. Chan JF, Yuan S, Kok KH, To KK, Chu H, Yang J et al. A fa- milial cluster of pneumonia associated with the 2019 novel coronavi- rus indicating person-to-person transmission: a study of a family clus- ter. Lancet 2020; 395 (10223): 514-523. DOI: 10.1016/s0140-6736 (20)30154-9.

12. Loeffelholz MJ, Tang YW. Laboratory diagnosis of emerging human coronavirus infections - the state of the art. Emerg Microbes Infect 2020; 9 (1): 747-756. DOI: 10.1080/22221751.2020.1745095.

13. Danese S, Sands B, Ng SC, Peyrin-Biroulet L. The day after COVID-19 in IBD: how to go back to 'normal'. Nat Rev Gastroenterol Hepatol 2020: 1-3. DOI: 10.1038/s41575-020-0322-8.

14. Jones L, Walsh K, Willcox M, Morgan P, Nichols J. The COVID-19 pandemic: Important considerations for contact lens practitioners. Cont Lens Anterior Eye 2020; 43 (3): 196-203. DOI: 10.1016/j. clae.2020.03.012.

15. Wölfel R, Corman VM, Guggemos W, Seilmaier M, Zange S, Müller MA et al. Virological assessment of hospitalized patients with COVID-2019. Nature 2020; 581 (7809): 465-469. DOI: 10.1038/s41586020-2196-x.

16. To KK, Tsang OT, Chik-Yan Yip C, Chan KH, Wu TC, Chan JMC et al. Consistent detection of 2019 novel coronavirus in saliva. Clin Infect Dis 2020. DOI: $10.1093 / \mathrm{cid} / \mathrm{ciaa} 149$.

17. Mallapaty S. Why does the coronavirus spread so easily between people? Nature 2020; 579 (7798): 183. DOI: 10.1038/d41586-020-00660-X.

18. Coutard B, Valle C, de Lamballerie X, Canard B, Seidah NG, Decroly E. The spike glycoprotein of the new coronavirus 2019-nCoV con- tains a furin-like cleavage site absent in $\mathrm{CoV}$ of the same clade. Antiviral Res 2020; 176: 104742. DOI: 10.1016/j.antiviral.2020.104742.

19. Izaguirre G. The Proteolytic Regulation of Virus Cell Entry by Furin and Other Proprotein Convertases. Viruses 2019; 11 (9). DOI: 10.3390/ v11090837.

20. Xu R, Cui B, Duan X, Zhang P, Zhou X, Yuan Q. Saliva: potential diagnostic value and transmission of 2019-nCoV. Int J Oral Sci 2020; 12 (1): 11. DOI: 10.1038/s41368-020-0080-z.

21. Gautier JF, Ravussin Y. A New Symptom of COVID-19: Loss of Taste and Smell. Obesity (Silver Spring) 2020; 28 (5): 848. DOI: 10.1002/ oby. 22809 .

22. Sungnak W, Huang N, Bécavin C, Berg M, Queen R, Litvinukova $\mathbf{M}$ et al. SARS-CoV-2 entry factors are highly expressed in nasal epithelial cells together with innate immune genes. Nat Med 2020; 26 (5): 681-687. DOI: $10.1038 / \mathrm{s} 41591-020-0868-6$.

23. Matsuyama S, Nagata N, Shirato K, Kawase M, Takeda M, Taguchi F. Efficient activation of the severe acute respiratory syndrome coronavirus spike protein by the transmembrane protease TMPRSS2. J Virol 2010; 84 (24): 12658-12664. DOI: 10.1128/jvi.01542-10.

24. Zhou P, Yang XL, Wang XG, Hu B, Zhang L, Zhang W et al. A pneumonia outbreak associated with a new coronavirus of probable bat origin. Nature 2020; 579 (7798): 270-273. DOI: 10.1038/s41586-020-2012-7.

25. Hoffmann M, Kleine-Weber H, Schroeder S, Krüger N, Herrler T, Erichsen S et al. SARS-CoV-2 Cell Entry Depends on ACE2 and TMPRSS2 and Is Blocked by a Clinically Proven Protease Inhibitor. Cell 2020; 181 (2): 271-280.e8. DOI: 10.1016/j.cell.2020.02.052.

26. Bertram S, Heurich A, Lavender H, Gierer S, Danisch S, Perin P et al. Influenza and SARS-coronavirus activating proteases TMPRSS2 and HAT are expressed at multiple sites in human respiratory and gastrointestinal tracts. PLoS One 2012; 7 (4): e35876. DOI: 10.1371/journal. pone. 0035876 .

27. Xu H, Zhong L, Deng J, Peng J, Dan H, Zeng X et al. High expression of ACE2 receptor of 2019-nCoV on the epithelial cells of oral mucosa. Int J Oral Sci 2020; 12 (1): 8. DOI: 10.1038/s41368-020-0074-x.

28. https://www.worldometers.info/coronavirus/?utm_campaign= home Advegas1?/embed/fd0k_hbXWcQ. Date of access: 25 Jun 2020.

29. Wilder-Smith A, Freedman DO. Isolation, quarantine, social distancing and community containment: pivotal role for old-style public health measures in the novel coronavirus (2019-nCoV) outbreak. J Travel Med 2020; 27 (2). DOI: 10.1093/jtm/taaa020.

30. Greenhalgh T, Schmid MB, Czypionka T, Bassler D, Gruer L. Face masks for the public during the covid-19 crisis. Bmj 2020; 369:m1435. DOI: $10.1136 /$ bmj.m1435.

31. Fantini J, Di Scala C, Chahinian H, Yahi N. Structural and molecular modelling studies reveal a new mechanism of action of chloroquine and hydroxychloroquine against SARS-CoV-2 infection. Int J Antimicrob Agents 2020; 55 (5): 105960. DOI: 10.1016/j.ijantimicag.2020.105960.

32. Lythgoe MP, Middleton P. Ongoing Clinical Trials for the Management of the COVID-19 Pandemic. Trends Pharmacol Sci 2020; 41 (6): 363-382. DOI: 10.1016/j.tips.2020.03.006.

33. McKee DL, Sternberg A, Stange U, Laufer S, Naujokat C. Candidate drugs against SARS-CoV-2 and COVID-19. Pharmacol Res 2020; 157: 104859. DOI: 10.1016/j.phrs.2020.104859.

34. Liu C, Zhou Q, Li Y, Garner LV, Watkins SP, Carter LJ et al. Research and Development on Therapeutic Agents and Vaccines for 
CO- VID-19 and Related Human Coronavirus Diseases. ACS Cent Sci 2020; 6 (3): 315-331. DOI: 10.1021/acscentsci.0c00272.

35. Youngren-Ortiz SR, Gandhi NS, España-Serrano L, Chougule MB. Aerosol Delivery of siRNA to the Lungs. Part 1: Rationale for Gene Delivery Systems. Kona 2016; 33: 63-85. DOI: 10.14356/kona. 2016014.

36. Ren Y, Kang CS, Yuan XB, Zhou X, Xu P, Han L et al. Co-delivery of as-miR-21 and 5-FU by poly (amidoamine) dendrimer attenuates human glioma cell growth in vitro. J Biomater Sci Polym Ed 2010; 21 (3): 303-314. DOI: $10.1163 / 156856209 \times 415828$.

37. Watts JK, Deleavey GF, Damha MJ. Chemically modified siRNA: tools and applications. Drug Discov Today 2008; 13 (19-20): 842-855. DOI: 10.1016/j.drudis.2008.05.007.

38. Gandhi NS, Tekade RK, Chougule MB. Nanocarrier mediated delivery of siRNA/miRNA in combination with chemotherapeutic agents for cancer therapy: current progress and advances. J Control Release 2014; 194: 238-256. DOI: 10.1016/j.jconrel.2014.09.001.

39. Youngren-Ortiz SR, Gandhi NS, España-Serrano L, Chougule MB. Aerosol Delivery of siRNA to the Lungs. Part 2: Nanocarrier-based Delivery Systems. Kona 2017; 34: 44-69. DOI: 10.14356/ kona.2017005.

40. Thomas CE, Ehrhardt A, Kay MA. Progress and problems with the use of viral vectors for gene therapy. Nat Rev Genet 2003; 4 (5): 346-358. DOI: $10.1038 / \mathrm{nrg} 1066$.

41. Tatiparti K, Sau S, Kashaw SK, Iyer AK. siRNA Delivery Strategies: A Comprehensive Review of Recent Developments. Nanomaterials (Basel) 2017; 7 (4). DOI: 10.3390/nano7040077.
42. Adams D, Gonzalez-Duarte A, O'Riordan WD, Yang CC, Ueda M, Kristen AV et al. Patisiran, an RNAi Therapeutic, for Hereditary Transthyretin Amyloidosis. N Engl J Med 2018; 379 (1): 11-21. DOI: 10.1056/ NEJMoa1716153.

43. Saw PE, Song EW. siRNA therapeutics: a clinical reality. Sci China Life Sci 2020; 63 (4): 485-500. DOI: 10.1007/s11427-018-9438-y.

44. Pan M, Ni J, He H, Gao S, Duan X. New paradigms on siRNA lo- cal application. BMB Rep 2015; 48 (3): 147-152. DOI: 10.5483/bmbrep.2015.48.3.089.

45. Vicentini FT, Borgheti-Cardoso LN, Depieri LV, de Macedo Mano D, Abelha TF, Petrilli R et al. Delivery systems and local administration routes for therapeutic siRNA. Pharm Res 2013; 30 (4): 915-931. DOI: 10.1007/s11095-013-0971-1.

46. Wu CJ, Huang HW, Liu CY, Hong CF, Chan YL. Inhibition of SARS-CoV replication by siRNA. Antiviral Res 2005; 65 (1): 45-48. DOI: 10.1016/j.antiviral.2004.09.005.

47. https://www.nature.com/articles/d41587-020-00005-z. 06.05.2020.

48. Kandil R, Merkel OM. Pulmonary delivery of siRNA as a novel treat- ment for lung diseases. Ther Deliv 2019; 10 (4): 203-206. DOI: 10.4155/ tde-2019-0009.

49. Pires A, Fortuna A, Alves G, Falcão A. Intranasal drug delivery: how, why and what for? J Pharm Pharm Sci 2009; 12 (3): 288-311. DOI: $10.18433 / j 3 n c 79$.

50. Hua S. Advances in Nanoparticulate Drug Delivery Approaches for Sublingual and Buccal Administration. Front Pharmacol 2019; 10: 1328. DOI: $10.3389 /$ fphar.2019.01328. 